\title{
A SPLITTING THEOREM FOR MANIFOLDS AND SURGERY GROUPS
}

\author{
BY SYLVAIN CAPPELL
}

Communicated by William Browder, December 19, 1969

I. In this paper we state a splitting theorem for manifolds and describe some applications and consequences. We announced a weaker form of this result in August 1969 at the Georgia Topology Conference. Besides its immediate applications, especially in the classification of homotopy equivalent manifolds, it implies the existence of a Mayer-Vietoris sequence for surgery groups. Using this, many surgery groups can be systematically computed and more results on the classification of manifolds can be obtained. Details of the proofs and applications will be published elsewhere.

This research was begun while I was working on my dissertation under the supervision of William Browder. His counsel and encouragement were invaluable and are deeply appreciated. Conversations with Friedhelm Waldhausen were also very useful.

By manifold we will mean either a differentiable, piecewise linear or topological manifold. Where no restrictions are stated, all the propositions and terminology of this paper can be interpreted in any of these three contexts.

Let $X^{n}$ be a closed codimension one submanifold of $Y^{n+1}$, a closed manifold, and $f: W \rightarrow Y$ a homotopy equivalence of closed manifolds. We call $f$ "splittable" if it is homotopic to a map, which we will continue to call $f$, such that $f$ is transverse regular to $X$ and $f \mid f^{-1} X \rightarrow X$ and $f \mid\left(W-f^{-1} X\right) \rightarrow Y-X$ are homotopy equivalences. If $Y-X$ has two connected components, we will call them $Y_{1}$ and $Y_{2}$ and if it has one component we will let $Y_{1}=Y-X$ and $Y_{2}=\varnothing$.

We call an inclusion of a group $H$ in a group $G$ two-sided if the only double coset $H z H, z \in H$, equal to its inverse, $H z^{-1} H$, is the trivial double coset. For $H$ normal in $G$ this means $G / H$ has no 2-torsion. If $G$ is finite, $H$ is two-sided in $G$ if, and only if, $H$ contains all the 2primary elements of $G$. In particular, any subgroup of a finite group of odd order is two-sided. If $\pi_{1}(X) \rightarrow \pi_{1}(Y)$ is an inclusion, it is two-

AMS 1970 subject classifications. Primary 57C10, 57D65, 57A35, 57C35, 57D35, 18F25; Secondary 57B10, 16A54, $20 \mathrm{H} 25$.

Key words and phrases. Classification of manifolds, surgery theory, surgery group, homotopy equivalent manifolds, submanifolds, Whitehead group, algebraic $K$-theory. 
sided if and only if both inclusions of $\pi_{1}(X)$ into $\pi_{1}(Y-X)$ are twosided.

THEOREM 1. Let $f: W \rightarrow Y$ be a homotopy equivalence of closed manifolds of dimension $(n+1)$ with $n \geqq 5$ and let $X \subset Y$ be a codimension one submanifold. Assume that

(i) $X$ is 2-sided in $Y$,

(ii) $\pi_{1} X \rightarrow \pi_{1} Y$ is injective.

(iii) $\pi_{1} X$ is 2-sided in $\pi_{1} Y$.

Then $f$ is splittable if and only if the Whitehead torsion of $f, \tau(f)$ $\in \mathrm{Wh}\left(\pi_{1} Y\right)$ is in the image of the natural map $\mathrm{Wh}\left(\pi_{1} Y_{1}\right) \oplus \mathrm{Wh}\left(\pi_{1} Y_{2}\right)$ $\rightarrow \mathrm{Wh}\left(\pi_{1} Y\right)$.

Let $N(X)$ denote an open neighborhood of $X$ in $Y$ homeomorphic to $X \times(0,1) \subset X \times[0,1] \subset Y$ and similarly for $N\left(f^{-1} X\right)=f^{-1}(X)$ $\times(0,1) \subset W$. Then the conclusion of Theorem 1 asserts that $f$, after modification by a homotopy, will induce homotopy equivalences of manifold pairs

$$
\left(\left(W-N\left(f^{-1} X\right)\right), \partial\left(W-N\left(f^{-1} X\right)\right)\right) \rightarrow(Y-N(X), \partial(Y-N(X))) .
$$

The kernel and cokernel of $\mathrm{Wh}\left(\pi_{1} Y_{1}\right) \oplus \mathrm{Wh}\left(\pi_{1} Y_{2}\right) \rightarrow \mathrm{Wh}\left(\pi_{1} Y\right)$ have been determined by $F$. Waldhausen [1]. We use in the proof of Theorem 1 , and in the statement of Theorem 2, a natural map $\phi: \operatorname{Wh}\left(\pi_{1} Y\right)$ $\rightarrow K_{0}\left(\pi_{1} X\right)$ defined by Waldhausen under the hypothesis of Theorem 1 .

Theorem 2. Hypotheses as in Theorem 1. Then if $\phi(\tau(f))=0$, there exists an h-cobordism $\left(V ; W, W^{\prime}\right)$ with $f$ extending to $F: V \rightarrow Y$ and with $F \mid W^{\prime}$ splittable.

Analogous results to Theorems 1 and 2 are true for manifolds with boundary. If either hypothesis (i) or (ii) above are removed, we can construct counterexamples to the desired conclusion. Of course, if $X$ and $Y$ are orientable condition (i) is always satisfied. In all of these results we do not actually need that $X$ and $Y$ are manifolds and the hypothesis can be restated in terms of Poincaré complexes. Without hypothesis (iii) in Theorem 2, we can show that $f$ is normally cobordant to a splittable map.

The Farrell-Hsiang splitting theorem [2] is the special case of Theorem 1, in which $Y-X$ is connected and $\pi_{1} Y=Z \times_{\alpha} \pi_{1} M$. In a private communication, $W$. Hsiang has informed me that he and Swarup have recently jointly proved Theorem 1 , in the case in which $n$ is even, and in the case $n$ odd with some additional hypothesis.

In the case in which $M$ cuts $W$ into two components, the first results were obtained by W. Browder [3]. He proved Theorem 1, in 
the case $\pi_{1} X=\pi_{1} Y=0$ together with some additional hypothesis. Wall proved a splitting theorem in the case in which $X$ cuts $Y$ into two components $Y_{1}$ and $Y_{2}$ and $\pi_{1} X=\pi_{1} Y_{1}$ and hence $\pi_{1}(Y)=\pi_{1}\left(Y_{2}\right)$ [4]. Ronnie Lee proved Theorem 1 in the case $n$ even and $\pi_{1}=0$ and $Y-X$ has two components [5].

Theorem 3. Let $f: W \rightarrow Y$ be a homotopy equivalence of P.L. or topological manifolds of dimension greater than 4 with $\pi_{1}(Y)$ having no elements of order 2 . If $Y$ is a connected sum of manifolds, $Y=P \# Q$, then $W$ is a connected sum $W=P^{\prime} \# Q^{\prime}$ with $f$ inducing homotopy equivalences of $P$ with $P^{\prime}$ and of $Q$ with $Q^{\prime}$.

PROof. For dimension greater than 5 , this is a special case of Theorem 1. Using this and Wall's periodicity theorem for surgery groups the result also follows in dimension 5 .

From the splitting theorem, we can obtain directly the following form of the Farrell fibering theorem [7]. The only result about Whitehead groups needed in the proof is the short exact sequence $\mathrm{Wh}(G) \stackrel{1-\alpha_{*}}{\rightarrow} \mathrm{Wh}(G) \rightarrow \mathrm{Wh}\left(Z \times_{\alpha} G\right)$. This is of course trivial for $\alpha=1$ and there is a simple geometric proof of this fact in the general case [7].

THEOREM 4. Let $W^{w+1} n \geqq 5$ be a manifold with $f: W \rightarrow S^{1}$ a continuous map with $f_{*}: \pi_{1}(w) \rightarrow \pi_{1}\left(S^{1}\right)$ onto. Then $f$ is homotopic to a fibration if and only if

(i) $\bar{W}$, the cover of $W$ associated to $\operatorname{Ker}\left(f_{*}\right) \subset \pi_{1} W$, has the homotopy type of a finite complex, and

(ii) an obstruction in $\mathrm{Wh}\left(\pi_{1} W\right)$ is zero.

OUTLINE OF PROOF. Using (i), we can construct a homotopy equivalence $g: W \rightarrow Y$ where $Y$ is a Poincaré complex which "homotopy fibres" over $S^{1}$ with fibre $X \subset Y$, a finite complex homotopy equivalent to $\bar{W}$. Then $\tau\left(g^{-1}\right) \in \mathrm{Wh}\left(\pi_{1} Y\right)$ will not depend upon the choices made. If it is 0 , we can split to get $M \subset W$ a candidate for a fibre and it readily follows that there is an $h$-cobordism $\left(V ; M, M^{\prime}\right)$ with $V \subset W$ and $M^{\prime}$ such that $W$ fibres with fibre $M^{\prime}$.

II. Let $L_{n}(\pi)$ denote the surgery obstruction group of $\pi$ for the homotopy equivalence problem as defined by C. T. C. Wall [4]. Let $\tilde{L}_{n}(\pi)$, the reduced surgery group of $\pi$, be the kernel of the natural map $L_{n}(\pi) \rightarrow L_{n}(0)$ so that $L_{n}(\pi)=\tilde{L}_{n}(\pi) \oplus L_{n}(0)$. Similarly let $L_{n}^{s}(\pi)$ denote the surgery obstruction group of $\pi$ for obtaining simple homotopy equivalences and, as above, $L_{n}^{s}(\pi)=\tilde{L}_{n}^{s}(\pi) \oplus L_{n}(0)$.

In what follows all groups $G_{i}, H_{i}$ and $K$ are assumed to have finite 
presentations. Let $G_{1} * G_{2}$ denote the free product of two groups. The following is a consequence of Theorem 3 .

TheOREM 5. If $G_{1}$ and $G_{2}$ have no elements of order 2,

$$
\begin{aligned}
& \tilde{L}_{n}\left(G_{1} * G_{2}\right)=\tilde{L}_{n}\left(G_{1}\right) \oplus \tilde{L}_{n}\left(G_{2}\right), \\
& \tilde{L}_{n}^{s}\left(G_{1} * G_{2}\right)=\tilde{L}_{n}^{s}\left(G_{1}\right) \oplus \tilde{L}_{n}^{*}\left(G_{2}\right) .
\end{aligned}
$$

For $n$ even, Theorem 5 was first proved by Ronnie Lee [5]. He also obtained the following corollary for the case $L_{4 k}$ and $L_{4 k+2}$.

CoROllary 6. Let $F_{m}$ denote the free group on $m$ generators. Then,

$$
L_{4 k}\left(F_{m}\right)=Z, \quad L_{4 k+1}\left(F_{m}\right)=Z^{m}, \quad L_{4 k+2}\left(F_{m}\right)=Z_{2}, \quad L_{4 k+3}\left(F_{m}\right)=Z_{2}^{m} .
$$

TheOREM 7. Let $H \subset G_{1}$ and $H \subset G_{2}$ be groups with both inclusions 2-sided or equivalently $H$ 2-sided in $G_{1} *_{H} G_{2}$ then

(i) If $\mathrm{Wh}(H) \rightarrow \mathrm{Wh}\left(G_{1}\right) \oplus \mathrm{Wh}\left(G_{2}\right)$ is 0 , then there is a long exact sequence

$$
\cdots \rightarrow L_{n}(H) \rightarrow L_{n}^{*}\left(G_{1}\right) \oplus L_{n}^{s}\left(G_{2}\right) \rightarrow L_{n}^{*}\left(G_{1} *_{H} G_{2}\right) \stackrel{\partial}{\rightarrow} L_{n-1}(H) \rightarrow \cdots
$$

(ii) If $K_{0}(H) \rightarrow K_{0}\left(G_{1}\right) \oplus K_{0}\left(G_{2}\right)$ is injective, there is a long exact sequence

$$
\cdots \rightarrow L_{n}(H) \rightarrow L_{n}\left(G_{1}\right) \oplus L_{n}\left(G_{2}\right) \rightarrow L_{n}\left(G_{1} *_{H} G_{2}\right) \stackrel{\partial}{\rightarrow} L_{n-1}(H) \rightarrow \cdots
$$

In the general case in which no special assumptions are made about $\mathrm{Wh}(H)$ and $K_{0}(H)$ we must introduce a new kind of surgery group $L^{\prime}\left(G_{1} *_{H} G_{2}\right)$. This will be the surgery obstruction group to obtaining homotopy equivalences whose Whitehead torsion lies in the image of $\mathrm{Wh}\left(G_{1}\right) \oplus \mathrm{Wh}\left(G_{2}\right) \rightarrow \mathrm{Wh}\left(G_{1} *_{H} G_{2}\right)$. There are natural maps $L^{\varepsilon}\left(G_{1} *_{H} G_{2}\right) \rightarrow L^{\prime}\left(G_{1} *_{H} G_{2}\right) \rightarrow L\left(G_{1} *_{H} G_{2}\right)$ and Rothenberg has shown that the composite map $L^{*}(G) \rightarrow L(G)$ has kernel and cokernel consisting of 2-torsion [6].

THEOREM 8. Let $H \subset G_{1}$ and $H \subset G_{2}$ be groups with both inclusions 2-sided or equivalently $H$ 2-sided in $G_{1} *_{H} G_{2}$. Then there is a long exact sequence

$$
\cdots \rightarrow L_{n}(H) \rightarrow L_{n}\left(G_{1}\right) \oplus L_{n}\left(G_{2}\right) \rightarrow L_{n}^{\prime}\left(G_{1} *_{H} G_{2}\right) \stackrel{\partial}{\rightarrow} L_{n-1}(H) \rightarrow \cdots
$$

and a long exact sequence

$$
\cdots \rightarrow B_{n+1} \rightarrow L_{n}^{\prime}\left(G_{1} *_{H} G_{2}\right) \rightarrow L_{n}\left(G_{1} *_{H} G_{2}\right) \rightarrow B_{n} \rightarrow \cdots
$$

with $B_{n}$ abelian and every element in it of order 2. 
Let $H \subset K$ and $H^{\prime} C K$ be subgroups of $K$ with $\alpha: H \rightarrow H^{\prime}$ an isomorphism. Let $t$ denote a generator of the integers $Z$ and let $G$ denote the group $Z * K /\left\{\alpha(x)=t x t^{-1}\right.$ for $\left.x \in H\right\}$.

Theorem 9. Assume $H$ and $H^{\prime}$ are 2-sided in $K$ or equivalently $H$ is 2-sided in G. (i) If $\mathrm{Wh}(H) \stackrel{1-\alpha_{*}}{\longrightarrow} \mathrm{Wh}(K)$ is 0 , there is a long exact sequence

$$
\cdots \rightarrow L_{n}(H) \stackrel{\left(1-\alpha_{*}\right)}{\longrightarrow} L_{n}^{s}(K) \rightarrow L_{n}^{s}(G) \stackrel{\partial}{\rightarrow} L_{n-1}(H) \rightarrow \cdots .
$$

(ii) If $\tilde{K}_{0}(H) \stackrel{\left(1-\alpha_{*}\right)}{\longrightarrow} \tilde{K}_{0}(K)$ is injective, there is a long exact sequence $\cdots \rightarrow L_{n}(H) \stackrel{\left(1-\alpha_{*}\right)}{\longrightarrow} L_{n}(K) \rightarrow L_{n}(G) \stackrel{\partial}{\rightarrow} L_{n-1}(H) \rightarrow \cdots$.

In the general case in which no special assumptions are made, we have the following result.

TheOREM 10. Assume $H$ and $H^{\prime}$ are 2-sided in $K$ or equivalently $H$ is 2-sided in $G$. There is a long exact sequence

$$
\cdots \rightarrow L_{n}(H) \stackrel{\left(1-\alpha_{*}\right)}{\longrightarrow} L_{n}(K) \rightarrow L_{n}^{\prime}(G) \stackrel{\partial}{\rightarrow} L_{n-1}(H) \rightarrow \cdots .
$$

Again $L_{n}^{\prime}(G)$ will be isomorphic to $L_{n}(G)$ "modulo 2-torsion". For $H=H^{\prime}=K$, Theorems 9 and 10 describe $L_{n}\left(Z \times_{\alpha} K\right)$, a description of which, assuming something about Whitehead groups, has already been obtained by Farrell and Hsiang [2]. For $Z \times K$ we obtain from Theorem 9, an exact sequence $0 \rightarrow L_{n}^{s}(K) \rightarrow L_{n}^{s}(Z \times K) \rightarrow L_{n-1}(K) \rightarrow 0$, and we obtain, using the natural splitting $L_{n}^{s}(Z \times K) \rightarrow L_{n}^{s}(K)$, a theorem of Shaneson and of Wall [4], [6], $L_{n}^{s}(Z \times K)=L_{n}^{s}(K) \oplus L_{n-1}(K)$.

We are forced to introduce the groups $L_{n}^{\prime}$ by the fact that not every homotopy equivalence is splittable and that even simple homotopy equivalences, when split, can produce homotopy equivalences which are not simple. All of the above results can be interpreted in the unoriented case by regarding $L_{n}$ as a functor of pairs $(\pi, \omega)$ with $\pi$ a finitely presented group and $\omega: \pi \rightarrow Z_{2}$ a homomorphism.

Geometrically, Theorem 7, corresponds to the case of the splitting theorem in which $Y-X$ has two components and Theorem 9 , to the case in which $Y-X$ has one component.

ADDED IN PROOF. In some cases we can show that hypothesis (iii) of Theorems 1 and 2 is unnecessary and thereby improve all the subsequent results. Under the hypothesis of Theorem 2, we can define a refined obstruction in $H^{n+1}\left(Z_{2} ; \operatorname{Ker}\left(\tilde{K}_{0}\left(Z\left[\pi_{1} X\right]\right) \rightarrow \tilde{K}_{0}\left(Z\left[\pi_{1}(Y-X)\right]\right)\right)\right)$ which is zero if and only if $f$ is $h$-cobordant to a splittable map. The hypothesis of all exact sequences stated above can be relaxed by using 
similar refined obstructions and the existence of a number of similar exact sequences can be demonstrated. Theorems 1 and 2 can also be formulated for open manifolds. The results and methods of this paper can be used to study duality in Waldhausen's decomposition of surgery groups and to obtain computations of $H^{n}\left(Z_{2} ; \mathrm{Wh}(G)\right)$ for $G=G_{1} *_{H} G_{2}$ or related groups.

\section{BIBLIOGRAPHY}

1. F. Waldhausen, Whitehead groups of generalized free products (to appear).

2. F. Farrell and W. Hsiang, On manifolds with $\pi_{1}=G \times_{\alpha} T_{r}$ Yale University, Notes, New Haven, Conn.

3. W. Browder, Embedding 1-connected manifolds, Bull. Amer. Math. Soc. 72 (1966), 225-231; Erratum, ibid., 72 (1966), 736. MR 32 \#6467.

4. C. T. C. Wall, Surgery of compact manifolds (to appear).

5. R. Lee, Splitting a manifold into two parts, Lecture notes, Institute for Advanced Study, Princeton, N. J., 1968.

6. - Wall's surgery obstruction groups for $G \times Z$, Ann. of Math. (2) 90 (1969), 296-334.

7. F. Farrell, The obstruction to fibering a manifold over a circle, Yale University Notes, New Haven, Conn., 1967.

Princeton University, Princeton, New Jersey 08540 